Скопје, Македонија

\title{
SOME CHARACTERIZATIONS OF 2-INNER PRODUCT
}

\author{
RISTO MALCHESKI, KATERINA ANEVSKA, AND SAMOIL MALCHESKI
}

\begin{abstract}
The characterization of 2-inner product is an issue which is the focus of interest of many mathematicians. In this paper, several equivalent characterizations of 2-inner product, that are consequences of Theorem 2 ([12]) are discussed. Thus, the equivalence of generalizations of the Jordan and von Neumann ([7]) and also Frechet ([8]) classical results, are proven. Furthermore, the characterization of Hlawka, the characterization of D. S. Marinescu, M. Monea, M. Opincariu and M. Stroe ([4]) are proven as well.
\end{abstract}

\section{INTRODUCTION}

Let $L$ be a real vector space with dimension greater than 1 and $\|\cdot, \cdot\|$ be a real function on $L \times L$ such that:

a) $\|x, y\| \geq 0$, for all $x, y \in L$ and $\|x, y\|=0$ if and only if the set $\{x, y\}$ is linearly dependent,

b) $\|x, y\|=\|y, x\|$, for all $x, y \in L$,

c) $\|\alpha x, y\|=|\alpha| \cdot\|x, y\|$, for all $x, y \in L$ and for each $\alpha \in \mathbf{R}$, and

d) $\|x+y, z\| \leq\|x, z\|+\|y, z\|$, for all $x, y, z \in L$.

The function $\|\cdot, \cdot\|$ is said to be 2 -norm of $L$, and $(L,\|\cdot, \cdot\|)$ is said to be vector 2-normed space ([11]). The inequality in the axiom $d)$ is said to be parallelepiped inequality.

Let $n>1$ be a positive integer, $L$ be a real vector space, $\operatorname{dim} L \geq n$ and $(\cdot, \cdot \mid \cdot)$ be a real function over $L \times L \times L$ such that:

i) $(x, x \mid y) \geq 0$, for all $x, y \in L$ and $(x, x \mid y)=0$ if and only if $x$ and $y$ are linearly dependent,

ii) $(x, y \mid z)=(y, x \mid z)$, for all $x, y, z \in L$,

iii) $(x, x \mid y)=(y, y \mid x)$, for all $x, y \in L$,

iv) $(\alpha x, y \mid z)=\alpha(x, y \mid z)$, for all $x, y, z \in L$.and for each $\alpha \in \mathbf{R}$, and

v) $\left(x+x_{1}, y \mid z\right)=(x, y \mid z)+\left(x_{1}, y \mid z\right)$, for all $x_{1}, x, y, z \in L$.

The function $(\cdot, \cdot \mid \cdot)$ is said to be 2 -inner product, and $(L,(\cdot, \cdot \mid \cdot))$ is said to be 2-pre-Hilbert space ([4]).

2000 Mathematics Subject Classification. 46C50, 46C15, 46B20.

Key words and phrases. 2-norm, 2-inner product, parallelepiped equality . 
The concepts of 2-norm and 2-inner product are two dimensional analogies to the concepts of a norm and an inner product. R. Ehret proved ([9]) that if $(L,(\cdot, \cdot \mid \cdot))$ is a 2-pre-Hilbert space, then

$$
\|x, y\|=(x, x \mid y)^{1 / 2}
$$

for all $x, y \in L$, defines a 2-norm.

So, we get vector 2-normed space $(L,\|\cdot, \cdot\|)$ and moreover, for all $x, y, z \in$ $L$ the following equalities are satisfied:

$$
\begin{gathered}
(x, y \mid z)=\frac{\|x+y, z\|^{2}-\|x-y, z\|^{2}}{4} \\
\|x+y, z\|^{2}+\|x-y, z\|^{2}=2\left(\|x, z\|^{2}+\|y, z\|^{2}\right)
\end{gathered}
$$

The equality (3) is actually analogous to the parallelogram equality and it is called parallelepiped equality. Further, 2-normed space $L$ is 2-preHilbert space if and only if for all $x, y, z \in L$ the equality (3) holds true.

The following theorem gives one other elementary characterization of 2 inner product, i.e. we will prove the equivalence of generalizations of the classical results of Jordan and von Neumann ([7]) and Frechet ([8]).

Theorem 1. Let $(L,\|\cdot, \cdot\|)$ be a real 2 -normed space. Then, $L$ is a 2-pre-Hilbert space if and only if

$$
\begin{gathered}
\|x+y+z, u\|^{2}+\|x, u\|^{2}+\|y, u\|^{2}+\|z, u\|^{2}= \\
=\|x+y, u\|^{2}+\|y+z, u\|^{2}+\|z+x, u\|^{2}
\end{gathered}
$$

for all $x, y, z, u \in L$.

Proof. Let the equality (4) hold true, for all $x, y, z, u \in L$. For $z=-y$, and for each $x, y, u \in L$ it is true that

$$
\|x, u\|^{2}+\|x, u\|^{2}+\|y, u\|^{2}+\|-y, u\|^{2}=\|x+y, u\|^{2}+\|0, u\|^{2}+\|x-y, u\|^{2},
$$

i.e. the equality (3) holds true, therefore, $L$ is a 2-pre-Hilbert space. Conversely, let $(L,\|\cdot, \cdot\|)$ be a 2 -pre-Hilbert space. Then the equation of the parallelogram implies that for all $x, y, z, u \in L$ the following holds true

$$
\begin{aligned}
\| x+y & +z, u\left\|^{2}+\right\| x, u\left\|^{2}+\right\| y, u\left\|^{2}+\right\| z, u \|^{2}= \\
& =\frac{1}{2}\|2 x+y+z, u\|^{2}+\frac{1}{2}\|y+z, u\|^{2}+\frac{1}{2}\|y+z, u\|^{2}+\frac{1}{2}\|y-z, u\|^{2} \\
& =\frac{1}{2}\|2 x+y+z, u\|^{2}+\frac{1}{2}\|y-z, u\|^{2}+\|y+z, u\|^{2} \\
& =\frac{1}{2}\|(x+y)+(x+z), u\|^{2}+\|(x+y)-(x+z), u\|^{2}+\|y+z, u\|^{2} \\
& =\|x+y, u\|^{2}+\|y+z, u\|^{2}+\|z+x, u\|^{2},
\end{aligned}
$$

i.e. the equality (4) holds true. 


\section{CHARACTERIZATION OF 2-PRE-HILBERT SPACE}

The problem of characterization of 2-pre-Hilbert spaces, i.e. the necessary and sufficient conditions, the 2-normed spaces to be treated as 2-preHilbert space is of particular interest while studying the 2-normed spaces. Thus, in [5] characterization of 2-pre-Hilbert space is given by using EulerLagrange type of equality, in [7] is given characterization by using strictly convex norm with modulus $c$, and in [9] are given characterizations by using Mercer inequality for 2-normed space and its equivalent inequality. Furthermore, in [13] the following theorem is proven.

Theorem 2. ([12]). Let $(L,\|\cdot, \cdot\|)$ be a real 2-normed space. $L$ is a 2pre-Hilbert space if and only if the following condition is satisfied: If $n \geq 3$, $x_{1}, x_{2}, \ldots, x_{n}, z \in L$ and $a_{1}, a_{2}, \ldots, a_{n}$ are real numbers such that $\sum_{i=1}^{n} a_{i}=0$, then

$$
\left\|\sum_{i=1}^{n} a_{i} x_{i}, z\right\|^{2}=-\sum_{1 \leq i<j \leq n} a_{i} a_{j}\left\|x_{i}-x_{j}, z\right\|^{2}
$$

In the next consequence, by applying theorems 1 and 2 we will prove the following generalization of Hlawkas result.

Corollary 1. Let $(L,\|\cdot, \cdot\|)$ be a real 2-normed space. $L$ is a 2-preHilbert space if and only if for each $n \geq 2$ and for each $x_{1}, x_{2}, \ldots, x_{n}, z \in L$ the following equality holds true

$$
\left\|\sum_{i=1}^{n} x_{i}, z\right\|^{2}+(n-2) \sum_{i=1}^{n}\left\|x_{i}, z\right\|^{2}=\sum_{1 \leq i<j \leq n}\left\|x_{i}+x_{j}, z\right\|^{2} .
$$

Proof. Let for each $n \geq 2$ and for all $x_{1}, x_{2}, \ldots, x_{n}, z \in L$ the equality (6) holds true. For $n=3$ and $x_{1}=x, x_{2}=y, x_{3}=u$ the equality (6) is transformed as the following:

$$
\begin{gathered}
\|x+y+u, z\|^{2}+\|x, z\|^{2}+\|y, z\|^{2}+\|u, z\|^{2}= \\
=\|x+y, z\|^{2}+\|y+u, z\|^{2}+\|u+x, z\|^{2},
\end{gathered}
$$

$x, y, u, z \in L$

by applying Theorem 1 we get that $L$ is a 2 -pre-Hilbert space.

Conversely, let $L$ be a 2 -pre-Hilbert space, $n \geq 2$ and $x_{1}, x_{2}, \ldots, x_{n}, z$ are arbitrary vectors from $L$. If we get that $a_{n+1}=-n, a_{i}=1, i=1,2, \ldots, n$ and $x_{n+1}=0$ then, by applying the above theorem and the parallelepiped equality the following equality holds true 


$$
\begin{aligned}
& \left\|\sum_{i=1}^{n} x_{i}, z\right\|^{2}=\left\|\sum_{i=1}^{n} x_{i}-n x_{n+1}, z\right\|^{2}= \\
& =n \sum_{i=1}^{n}\left\|x_{i}-x_{n+1}, z\right\|^{2}-\sum_{1 \leq i<j \leq n}\left\|x_{i}-x_{j}, z\right\|^{2} \\
& \left.=n \sum_{i=1}^{n}\left\|x_{i}, z\right\|^{2}-2 \sum_{1 \leq i<j \leq n}\left(\left\|x_{i}, z\right\|^{2}+\left\|x_{j}, z\right\|^{2}\right)+\sum_{1 \leq i<j \leq n}\left\|x_{i}+x_{j}, z\right\|^{2}\right) \\
& \left.\quad=n \sum_{i=1}^{n}\left\|x_{i}, z\right\|^{2}-2(n-1) \sum_{i=1}^{n}\left\|x_{i}, z\right\|^{2}+\sum_{1 \leq i<j \leq n}\left\|x_{i}+x_{j}, z\right\|^{2}\right),
\end{aligned}
$$

which is equivalent to equality (6).

In the following considerations will be proven several consequences of Theorem 2, which are actually generalizations of the results given by D. S. Marinescu, M. Monea, M. Opincariu and M. Stroe ([4]).

Corollary 2. Let $(L,\|\cdot, \cdot\|)$ be a real 2-normed space. $L$ is a 2-preHilbert space if and only if for each $n \geq 2$, for all $a_{1}, a_{2}, \ldots, a_{n} \in \mathbf{R}$ and for all $x_{1}, x_{2}, \ldots, x_{n}, z \in L$ the following equality holds true

$$
\begin{gathered}
\left\|\sum_{i=1}^{n} a_{i} x_{i}, z\right\|^{2}= \\
=\left(a_{1}+a_{2}+\ldots+a_{n}\right) \sum_{i=1}^{n} a_{i}\left\|x_{i}, z\right\|^{2}-\sum_{1 \leq i<j \leq n} a_{i} a_{j}\left\|x_{i}-x_{j}, z\right\|^{2} .
\end{gathered}
$$

Proof. For each $n \geq 2$, and for all $a_{1}, a_{2}, \ldots, a_{n} \in \mathbf{R}$ and $x_{1}, x_{2}, \ldots, x_{n}, z \in$ $L$, let the equality (7) hold true. For $n=2, a_{1}=a_{2}=1, x_{1}=x, x_{2}=y$ the equality (7) is transformed as the following

$$
\|x+y, z\|^{2}=2\left(\|x, z\|^{2}+\|y, z\|^{2}\right)-\|x-y, z\|^{2}, x, y, z \in L
$$

i.e. the equality (3) holds true. The latter means that $L$ is a 2-pre-Hilbert space.

Conversely, let $L$ be a 2 -pre-Hilbert space, $n \geq 2, a_{1}, a_{2}, \ldots, a_{n}$ be arbitrary real numbers and $x_{1}, x_{2}, \ldots, x_{n}, z$ arbitrary vectors on $L$.

Let $a_{n+1}=-\left(a_{1}+a_{2}+\ldots+a_{n}\right)$ and $x_{n+1}=0$. Since Theorem 2 we get the following

$$
\begin{aligned}
\left\|\sum_{i=1}^{n} a_{i} x_{i}, z\right\|^{2} & =\left\|\sum_{i=1}^{n+1} a_{i} x_{i}, z\right\|^{2}=-\sum_{1 \leq i<j \leq n+1} a_{i} a_{j}\left\|x_{i}-x_{j}, z\right\|^{2} \\
& =-\sum_{i=1}^{n} a_{i} a_{n+1}\left\|x_{i}-x_{n+1}, z\right\|^{2}-\sum_{1 \leq i<j \leq n} a_{i} a_{j}\left\|x_{i}-x_{j}, z\right\|^{2} \\
& =-\left(a_{1}+a_{2}+\ldots+a_{n}\right) \sum_{i=1}^{n} a_{i}\left\|x_{i}, z\right\|^{2}-\sum_{1 \leq i<j \leq n} a_{i} a_{j}\left\|x_{i}-x_{j}, z\right\|^{2},
\end{aligned}
$$


The latter means that the equality (7) holds true.

Corollary 3. Let $(L,\|\cdot, \cdot\|)$ be a real 2-normed space. $L$ is a 2-preHilbert space if and only if for each $n \geq 2$, for all $a_{1}, a_{2}, \ldots, a_{n} \in \mathbf{R}$ such that $\sum_{i=1}^{n} a_{i}=1$ and for all $x_{1}, x_{2}, \ldots, x_{n}, z \in L$ the following equality holds true

$$
\left\|\sum_{i=1}^{n} a_{i} x_{i}, z\right\|^{2}=\sum_{i=1}^{n} a_{i}\left\|x_{i}, z\right\|^{2}-\sum_{1 \leq i<j \leq n} a_{i} a_{j}\left\|x_{i}-x_{j}, z\right\|^{2} .
$$

Proof. Let for each $n \geq 2$, for all $a_{1}, a_{2}, \ldots, a_{n} \in \mathbf{R}$ so that $\sum_{i=1}^{n} a_{i}=1$ and $x_{1}, x_{2}, \ldots, x_{n}, z \in L$, the equality (8) hold true. For $n=2, a_{1}=a_{2}=\frac{1}{2}$, $x_{1}=x, x_{2}=y$ the equality (8) is transformed as following

$$
\left\|\frac{1}{2} x+\frac{1}{2} y, z\right\|^{2}=\frac{1}{2}\|x, z\|^{2}+\frac{1}{2}\|y, z\|^{2}-\frac{1}{4}\|x+y, z\|^{2}
$$

for all $x, y, z \in L$. The latter is equivalent to the equation (3). Therefore $L$ is 2-pre-Hilbert space.

Conversely, let $L$ be a 2 -pre-Hilbert space and $n \geq 2, a_{1}, a_{2}, \ldots, a_{n}$ are any real numbers so that $\sum_{i=1}^{n} a_{i}=1$ and $x_{1}, x_{2}, \ldots, x_{n}, z$ are any vectors on $L$. Let $a_{n+1}=-1$ and $x_{n+1}=0$. Theorem 2 implies the following

$$
\begin{aligned}
\left\|\sum_{i=1}^{n} a_{i} x_{i}, z\right\|^{2} & =\left\|\sum_{i=1}^{n+1} a_{i} x_{i}, z\right\|^{2}=-\sum_{1 \leq i<j \leq n+1} a_{i} a_{j}\left\|x_{i}-x_{j}, z\right\|^{2} \\
& =-\sum_{i=1}^{n} a_{i} a_{n+1}\left\|x_{i}-x_{n+1}, z\right\|^{2}-\sum_{1 \leq i<j \leq n} a_{i} a_{j}\left\|x_{i}-x_{j}, z\right\|^{2} \\
& =\sum_{i=1}^{n} a_{i}\left\|x_{i}, z\right\|^{2}-\sum_{1 \leq i<j \leq n} a_{i} a_{j}\left\|x_{i}-x_{j}, z\right\|^{2},
\end{aligned}
$$

The latter means that (8) holds true.

Corollary 4. Let $(L,\|\cdot, \cdot\|)$ be a real 2-normed space. Then $L$ is a 2pre-Hilbert space if and only if for all $n \geq 2$, for each $a_{1}, a_{2}, \ldots, a_{n} \in \mathbf{R} \backslash\{0\}$ so that $\sum_{i=1}^{n} a_{i} \neq 0$ and for all $x_{1}, x_{2}, \ldots, x_{n}, z \in L$ the following equality holds true

$$
\sum_{i=1}^{n} \frac{\left\|x_{i}, z\right\|^{2}}{a_{i}}-\frac{1}{\sum_{i=1}^{n} a_{i}}\left\|\sum_{i=1}^{n} x_{i}, z\right\|^{2}=\frac{1}{\sum_{i=1}^{n} a_{i}} \sum_{1 \leq i<j \leq n} \frac{\left\|a_{j} x_{i}-a_{i} x_{j}, z\right\|^{2}}{a_{i} a_{j}}
$$


Proof. Let for each $n \geq 2$, for all $a_{1}, a_{2}, \ldots, a_{n} \in \mathbf{R} \backslash\{0\}$ so that $\sum_{i=1}^{n} a_{i} \neq 0$ and for all $x_{1}, x_{2}, \ldots, x_{n}, z \in L$ the equality (9) holds true. For $n=2$, $a_{1}=a_{2}=1, x_{1}=x, x_{2}=y$ the equality (9) is the following

$$
\|x, z\|^{2}+\|y, z\|^{2}-\frac{1}{2}|| x+y, z\left\|^{2}=\frac{1}{2}\right\| x-y, z \|^{2},
$$

for all $x, y, z \in L$. The latter is equivalent to (3). Thus, $L$ is 2 -pre-Hilbert space.

Conversely, let $L$ be a 2 -pre-Hilbert space and $n \geq 2, a_{1}, a_{2}, \ldots, a_{n} \in$ $\mathbf{R} \backslash\{0\}$ are arbitrary real numbers so that $\sum_{i=1}^{n} a_{i} \neq 0$ holds true and $x_{1}, x_{2}, \ldots$, $x_{n}, z$ are arbitrary vectors on $L$. Let $a_{n+1}=-1$ and $x_{n+1}=0$.

Theorem 2 implies the following

$$
\begin{aligned}
\left\|\sum_{i=1}^{n} a_{i} x_{i}, z\right\|^{2} & =\left\|\sum_{i=1}^{n+1} a_{i} x_{i}, z\right\|^{2}=-\sum_{1 \leq i<j \leq n+1} a_{i} a_{j}\left\|x_{i}-x_{j}, z\right\|^{2} \\
& =-\sum_{i=1}^{n} a_{i} a_{n+1}\left\|x_{i}-x_{n+1}, z\right\|^{2}-\sum_{1 \leq i<j \leq n} a_{i} a_{j}\left\|x_{i}-x_{j}, z\right\|^{2} \\
& =\sum_{i=1}^{n} a_{i}\left\|x_{i}, z\right\|^{2}-\sum_{1 \leq i<j \leq n} a_{i} a_{j}\left\|x_{i}-x_{j}, z\right\|^{2},
\end{aligned}
$$

The latter means that (9) holds true.

Corollary 5. Let $(L,\|\cdot, \cdot\|)$ be a real 2-normed space. $L$ is a 2-preHilbert space if and only if for each $n \geq 2$ and for all $x_{1}, x_{2}, \ldots, x_{n}, z \in L$ the following holds true

$$
\frac{1}{n}\left\|\sum_{i=1}^{n} x_{i}, z\right\|^{2}=\sum_{i=1}^{n}\left\|x_{i}, z\right\|^{2}-\frac{1}{n} \sum_{1 \leq i<j \leq n}\left\|x_{i}-x_{j}, z\right\|^{2} .
$$

Proof. Let (10) hold true for each $n \geq 2$ and for all $x_{1}, x_{2}, \ldots, x_{n}, z \in L$. For $x_{1}=x, x_{2}=y(10)$ is transformed as the following

$$
\frac{1}{2}\|x+y, z\|^{2}=\|x, z\|^{2}+\|y, z\|^{2}-\frac{1}{2}\|x-y, z\|^{2},
$$

for all $x, y, z \in L$. The above equality is equivalent to (3). Thus, $L$ is 2 -preHilbert space. Conversely, let $L$ be a 2 -pre-Hilbert space and $n \geq 2$ and $x_{1}, x_{2}, \ldots, x_{n}, z$ are arbitrary vectors on $L$. Let $a_{n+1}=-1, a_{i}=n, i=$ $1,2, \ldots, n$ and $x_{n+1}=0$. Theorem 2 implies the following 


$$
\begin{aligned}
\left\|\sum_{i=1}^{n} \frac{1}{n} x_{i}, z\right\|^{2} & =\left\|\sum_{i=1}^{n} \frac{1}{n} x_{i}-x_{n+1}, z\right\|^{2}= \\
& =\frac{1}{n} \sum_{i=1}^{n}\left\|x_{i}-x_{n+1}, z\right\|^{2}-\sum_{1 \leq i<j \leq n} \frac{1}{n^{2}}\left\|x_{i}-x_{j}, z\right\|^{2} \\
& =\frac{1}{n} \sum_{i=1}^{n}\left\|x_{i}, z\right\|^{2}-\frac{1}{n^{2}} \sum_{1 \leq i<j \leq n}\left\|x_{i}-x_{j}, z\right\|^{2},
\end{aligned}
$$

The latter is equivalent to (10).

\section{CONFLICT OF INTEREST}

No conflict of interest was declared by the author.

\section{References}

[1] C. Diminnie, A. White, A characterizatin of 2-inner product spaces, Math. Japon. No. 27 (1982), 357-377.

[2] C. Diminnie, S. Gahler, A. White, 2-Inner Product Spaces II, Demonstratio Mathematica, Vol. X, No 1 (1977), 169-188

[3] C. Diminnie, S. Gahler, A. White, 2-Inner Product Spaces, Demonstratio Mathematica, Vol. VI (1973), 525-536

[4] D. S. Marinescu, M. Monea, M. Opimcariu, M. Stroe, Some Equivalent Characterizations of Inner Product Spaces and Their Consequences, Filomat 29 (7) (2015), 1587-1599

[5] K. Anevska, R. Malceski, Characterization of 2-inner product using Euler-Lagrange type of equality, International Journal of Science and Research (IJSR), ISSN 2319-7064, Vol. 3 Issue 6 (2014), 1220-1222.

[6] M. Frechet, Sur la definition axiomatique dune classe despaces vectoriels distancies applicable vectorillement sur lespace de Hilbert, Ann. of Math. Vol. 36 (3) (1935), 705-718.

[7] P. Jordan, J. von Neumann, On inner products in linear, metric spaces, Ann. of Math. Vol. 36 (3) (1935), 719-723.

[8] R. Ehret, Linear 2-Normed Spaces, Doctoral Diss., Saint Louis Univ., 1969

[9] R. Malceski, K. Anevska, Characterization of 2-inner product by strictly convex 2-norm of modul c, International Journal of Mathematical Analysis, Vol. 8, no. 33 (2014), 1647-1652

[10] S. Gahler, Lineare 2-normierte Raume, Math. Nachr. 28 (1965), $1-42$

[11] S. Malceski, A. Malceski, K. Anevska, R. Malceski, Another characterizations of 2-pre-Hilbert space, IJSIMR, e-ISSN 2347-3142, p-ISSN 2346-304X, Vol. 3, Issue 2 (2015), pp. 45-54.

[12] S. Malceski, K. Anevska, R. Malceski, New characterization of 2pre-Hilbert Space, atematicki bilten, Vol. 38(XLIV) no. 1 (2014), 31-38

[13] Y. J. Cho, S. S. Kim, Gateaux derivatives and 2-Inner Product Spaces, Glasnik Math., Vol. 27 (47) (1992) , 271-282 
[14] Р. Малчески, Забелешки за $n$-нормирани простори, Matematicki bilten, Vol. 20 (1996), 33-50

[15] K. Anevska, R. Malceski, Remark about characterization of 2-inner product, atematicki bilten, Vol. 40(XLIV) no. 3 (2016), 29-36

Faculty of informatics, FOn University, Skopje, Macedonia.

E-mail address: risto.malceski@gmail.com

Faculty of informatics, FOn University, Skopje, Macedonia.

E-mail address: anevskak@gmail.com

University Euro-Balkan, Skopje, Macedonia.

E-mail address: samoil.malcheski@gmail.com 